\title{
Early-life disruption of epigenetic marks may contribute to the origins of mental illness
}

\author{
"Changes in DNA methylation during early childhood could partially explain the \\ discordance of psychiatric disease in monozygotic twins."
}

\section{KEYWORDS: anxiety $\approx$ biomarkers $\approx$ bipolar disorder $\approx$ depression $\approx$ DNA methylation = epigenetics $=$ post-traumatic stress disorder $\approx$ schizophrenia}

Epigenetic marks, including DNA methylation (DNAm), histone modifications and small inhibitory RNAs, are heritable and provide critical layers of gene regulatory control; however, they also are dynamic and can be influenced by environmental exposures [1-3]. Studies primarily focused on cancer and late-onset diseases have revealed that a growing number of human diseases are linked to epigenetic disruptions. For example, DNAm became a hallmark of human cancer, a link that was first recognized in 1983, when tumor cells were found to be aberrantly methylated compared with their normal cell counterparts due to a loss of methylation from repetitive regions of the genome [4]. Together with the finding that healthy monozygotic twins show indistinguishable childhood DNAm [5], epigenetic mechanisms have long been thought to be highly coordinated throughout early development and more susceptible to drift in adulthood. In this article, we will discuss the impact that recent findings, of dramatic DNAm changes throughout childhood, will have on future epigenetic studies conducted in children and adults.

\section{"Knowing the complete repertoire of effects that early-life adversity has on epigenetic marks will improve an individual's prognosis, diagnosis and treatment..."}

DNAm is the best understood epigenetic modification and it primarily occurs at cytosines located $5^{\prime}$ to guanines in $\mathrm{CpG}$ dinucleotides of differentiated eukaryotic cells [6]. This modification is less common in CpG-rich areas, known as $\mathrm{CpG}$ islands, which are often located in the promoter regions of many genes and function to repress transcription. In addition, DNAm plays a key role in chromatin structure, chromosome stability, genomic imprinting and the maintenance of the inactive $\mathrm{X}$ chromosome in females. DNAm has long been considered a stable epigenetic mark; however, molecular evidence indicates that it can vary during embryogenesis, the establishment of $\mathrm{X}$ inactivation and in stem cell lineage formation. To study 'normal' DNAm variation early in life, we examined nearly half a million $\mathrm{CpG}$ dinucleotides throughout the genomes of almost 400 boys aged 3-17 years and found significant changes in DNAm at nearly $10 \%$ of loci throughout the genome [7]. A preference for specific nucleotides immediately surrounding the interrogated $\mathrm{CpG}$ dinucleotide suggested that these changes are not stochastic, which was also evident by their primary association with developmental and immune ontological functions. Indeed, these are biologically relevant activities for this developmental period. Finally, these studies revealed a significant overlap between the pediatric age-associated loci and previously identified adult loci; however, the pediatric loci were changing at a much faster rate (three- to five-times), indicating a more robust age-associated DNAm variation in children than adults. These data challenge the dogma regarding the susceptibility of DNAm to drift throughout life, suggesting it is even more susceptible during early-life development, which may contribute to disease in children as well as adults.

Changes in DNAm during early childhood could partially explain the discordance of psychiatric disease in monozygotic twins. Despite their identical genomes, the development of mental illness varies between twin pairs, resulting in the following concordance rates: $37 \%$ for depression; $40 \%$ for bipolar disorder; $50 \%$ for schizophrenia; and up to $80 \%$ for attention deficit hyperactivity disorder [8-11]. Moreover, numerous groups have shown that early diagnosis and treatment of children with attention deficit hyperactivity disorder and autism has

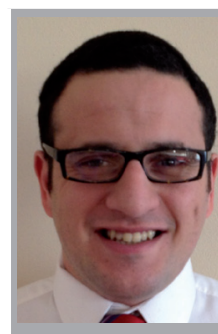

Elliot R Lee

Department of Psychiatry, University of Wisconsin - Madison, 6001 Research Park Boulevard, Madison, WI 53719, USA

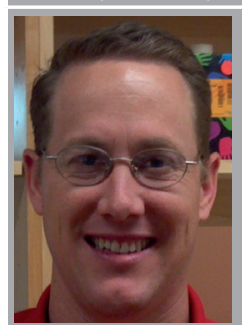

Reid S Alisch

Author for correspondence: Department of Psychiatry, University of Wisconsin-Madison, 6001 Research Park Boulevard, Madison, WI 53719, USA Tel.: +16082628430 alisch@wisc.edu 
profound effects on improving their outcome and quality of life. Thus, it is of great interest to determine the epigenetic marks associated with these diseases, and the environmental factors that cause them to vary, which could dramatically advance our diagnostic ability and potentially lead to the development of therapies aimed to prevent these modifications.

Studies in animal models often provide clues to improve our understanding of the effects of early-life adversity. One such study revealed that maternal grooming in rodents was inversely correlated with the DNAm level at the promoter of the glucocorticoid receptor gene, $N R 3 C 1$, resulting in altered $N R 3 C 1$ expression levels [12]. A consistent finding was shown in humans when suicide victims with a history of abuse or neglect have a similar relationship between life experiences, DNAm and $N R 3 C 1$ expression levels [13]. These data shed light on the potential role of DNA methylation in the development of pediatric disorders such as depression, suggesting that changes in DNAm are established presymptomatically due to early-life adversity. Knowing the complete repertoire of effects that early-life adversity has on epigenetic marks will improve an individual's prognosis, diagnosis and treatment, all of which are in need of advancement in the field of psychiatry.

“...schizophrenia is a neurodevelopmental disorder and ... epigenetic marks established early in life could provide a blueprint towards its pathogenesis."

Schizophrenia, which also presents later in life, may have a similar etiology that is initiated by perinatal insults, resulting in deficits in cortical maturation during childhood and adolescence [14]. Consistent with this notion, the RELN gene, which encodes a protein involved in long-term memory formation, has increased DNAm and reduced expression in schizophrenic patients [15]. In addition, while increased DNAm of the GAD1 promoter results in decreased expression of GAD1 in cortical tissues, reduced DNAm at the catechol- $O$-methyltransferase promoter contributes to alterations of the GABAergic circuitry [16]. Together, these findings are cultivating a perception that schizophrenia is a neurodevelopmental disorder and that epigenetic marks established early in life could provide a blueprint towards its pathogenesis. Consistent with this perception, a recent study found that the promoter region of $\alpha-N$ acetylgalactosaminide $\alpha-2,6$-sialyltransferase 1 was significantly differentially methylated in the peripheral blood of monozygotic twins discordant for schizophrenia and bipolar disorder [17]. Future studies that determine the developmental timing of these changes (i.e., presymptomatically and/or during childhood) will direct the diagnosis and treatment of these patients in the coming years.

\section{"...the likelihood of finding markers for early detection and diagnosis of molecularly less well-defined diseases seems within our reach."}

Finding roles for epigenetics in neuropsychiatric disease may reveal novel pathways for therapeutic drug development, primarily taking advantage of the fact that epigenetic modifications are reversible. For example DNA methyltransferase inhibitors such as 5-azacytidine reverse the hypermethylated marks at the RELN promoter in vitro, producing a 60 -fold increase in RELN mRNA levels [18]. Valproic acid, which is commonly used to treat bipolar disorder, has histone deacetylase inhibitor properties and has been shown to increase the expression of RELN and GAD1 in animal models [19]. Clearly these data suggest that molecular management of epigenetics marks associated with neuropsychiatric disorders has extreme therapeutic potential.

Epigenetics marks are used as biomarkers to molecularly diagnose diseases associated with disruption in genomic imprinting (e.g., Beckwith-Wiedemann syndrome), an approach that also could be adapted for psychiatric disorders. Since current psychiatric diagnoses are based on subjective criteria from the diagnostic and statistical manual [20], development of a less subjective tool is of great interest. Several groups have begun to explore these options and have had limited success in finding biological correlates for diseases such as depression, schizophrenia and bipolar disorder [21]. By contrast, a promising study recently found blood based biomarkers associated with Alzheimer's disease and standardized tests for these marks are currently under development [22]. As our understanding of epigenetic associations with disease improves, the likelihood of finding markers for early detection and diagnosis of molecularly less well-defined diseases seems within our reach.

Armed with the knowledge that epigenetic marks are susceptible to change early in life and throughout childhood, research can now focus on earlier molecular origins of mental illness. These studies may begin to discover marks that 
indicate either a predisposition or a resiliency to disorders that likely manifest very early in life following a stress related event, including depression, anxiety or post-traumatic stress disorder. Thus, the field of behavioral epigenetics is poised to alter our fundamental understanding of psychiatric disease, exposing the fact that the biopsychosocial formulation of a patient's illness will be correlated with the interaction of genes and environment.

\section{References}

1 Volpe TA, Kidner C, Hall IM, Teng G, Grewal SI, Martienssen RA. Regulation of heterochromatic silencing and histone $\mathrm{H} 3$ lysine- 9 methylation by RNAi. Science 297(5588), 1833-1837 (2002).

2 Saxonov S, Berg P, Brutlag DL. A genomewide analysis of $\mathrm{CpG}$ dinucleotides in the human genome distinguishes two distinct classes of promoters. Proc. Natl Acad. Sci. USA 103(5), 1412-1417 (2006).

3 Jenuwein T, Allis CD. Translating the histone code. Science 293(5532), 1074-1080 (2001).

4 Feinberg AP, Vogelstein B. Hypomethylation distinguishes genes of some human cancers from their normal counterparts. Nature 301(5895), 89-92 (1983).

5 Fraga MF, Ballestar E, Paz MF et al. Epigenetic differences arise during the lifetime of monozygotic twins. Proc. Natl Acad. Sci. USA 102(30), 10604-10609 (2005).

6 Jaenisch R, Bird A. Epigenetic regulation of gene expression: how the genome integrates intrinsic and environmental signals. Nat. Genet. 33(Suppl.), 245-254 (2003).

7 Alisch RS, Barwick BG, Chopra P et al. Age-associated DNA methylation in pediatric populations. Genome Res. 22(4), 623-632 (2012).

\section{Financial \& competing interests disclosure}

The authors have no relevant affliations or financial involvement with any organization or entity with a financial interest in or financial conflict with the subject matter or materials discussed in the manuscript. This includes employment, consultancies, honoraria, stock ownership or options, expert testimony, grants or patents received or pending, or royalties.

No writing assistance was utilized in the production of this manuscript.

8 Craddock N, Jones I. Genetics of bipolar disorder. J. Med. Genet. 36(8), 585-594 (1999).

9 Sullivan PF, Prescott CA, Kendler KS. The subtypes of major depression in a twin registry. J. Affect Disord. 68(2-3), 273-284 (2002).

10 Kringlen E. Twin studies in schizophrenia with special emphasis on concordance figures. Am. J. Med. Genet. 97(1), 4-11 (2000).

11 Levy F, Hay DA, McStephen M, Wood C, Waldman I. Attention-deficit hyperactivity disorder: a category or a continuum? Genetic analysis of a large-scale twin study. J. Am Acad. Child Adolesc. Psychiatry 36(6), 737-744 (1997).

12 Weaver IC, Cervoni N, Champagne FA et al. Epigenetic programming by maternal behavior. Nat. Neurosci. 7(8), 847-854 (2004).

13 McGowan PO, Sasaki A, D’Alessio AC et al. Epigenetic regulation of the glucocorticoid receptor in human brain associates with childhood abuse. Nat. Neurosci. 12(3), 342-348 (2009).

14 Brown AS, Patterson PH. Maternal infection and schizophrenia: implications for prevention. Schizophr. Bull. 37(2), 284-290 (2011).

15 Grayson DR, Jia X, Chen Y et al. Reelin promoter hypermethylation in schizophrenia. Proc. Natl Acad. Sci. USA 102(26), 9341-9346 (2005).
16 Abdolmaleky HM, Cheng KH, Faraone SV et al. Hypomethylation of MB-COMT promoter is a major risk factor for schizophrenia and bipolar disorder. Hum. Mol. Genet. 15(21), 3132-3145 (2006).

17 Dempster EL, Pidsley R, Schalkwyk LC et al. Disease-associated epigenetic changes in monozygotic twins discordant for schizophrenia and bipolar disorder. Hum. Mol. Genet. 20(24), 4786-4796 (2011).

18 Chen Y, Sharma RP, Costa RH, Costa E, Grayson DR. On the epigenetic regulation of the human reelin promoter. Nucleic Acids Res. 30(13), 2930-2939 (2002)

19 Tremolizzo L, Carboni G, Ruzicka WB et al. An epigenetic mouse model for molecular and behavioral neuropathologies related to schizophrenia vulnerability. Proc. Natl Acad. Sci. USA 99(26), 17095-17100 (2002).

20 American Psychiatric Association. Diagnostic and Statistical Manual of Mental Disorders (4th Edition Text Revision). American Psychiatric Association, DC, USA (2000).

21 Perlis RH. Translating biomarkers to clinical practice. Mol. Psychiatry 16(11), 1076-1087 (2011)

22 Mayeux R, Schupf N. Blood-based biomarkers for Alzheimer's disease: plasma $A \beta 40$ and $A \beta 42$, and genetic variants. Neurobiol. Aging 32(Suppl. 1), S10-S19 (2011) 